\title{
Tunable Load MIMO with Quantized Loads
}

\author{
Ang $\mathrm{Li}^{\dagger}$, Christos Masouros ${ }^{\dagger}$, Mathini Sellathurai ${ }^{\circ}$, and Constantinos B. Papadias* \\ Dept. of Electronic and Electrical Eng., University College London, London, U.K. ${ }^{\dagger}$ \\ Signal Process. and Commun. Research Group, Heriot-Watt University, EH14 4AS, U.K. ${ }^{\circ}$ \\ Broadband Wireless and Sensor Networks Group, Athens Information Technology, 19002 Athens, Greece* \\ Email: \{ang.li.14, c.masouros\}@ucl.ac.uk ${ }^{\dagger}$, M.Sellathurai@hw.ac.uk ${ }^{\circ}$,papadias@ait.edu.gr*
}

\begin{abstract}
In this paper, we study the application of precoding schemes on practical electronically steerable parasitic array radiators (ESPARs), where quantized load impedances are considered for each antenna element. The presence of quantization in the loads results in a performance loss for practical ESPARs. To alleviate the performance loss, we propose to approximate the ideal current vector with convex optimization, where it is further shown that the optimality is achieved by optimizing the feeding voltages only. Specifically, we obtain the closed-form expression when single-fed ESPARs are assumed. Numerical results show that the proposed quantization-robust scheme can achieve a significant performance gain over ESPARs with quantized loads.
\end{abstract}

Index Terms-MIMO, ESPARs, quantization, optimization.

\section{INTRODUCTION}

Multiple-input multiple-output (MIMO) systems have been widely acknowledged as a promising technology, and precoding techniques have been extensively studied for multi-user transmission [1]-[7]. The dirty paper coding (DPC) proposed in [1] can achieve the channel capacity by subtracting the interference before transmission, which is however difficult to implement in practice due to the complexity and the assumption of infinite codeword length. Non-linear precoding schemes such as Tomlinson-Harashima precoding (THP) [2] and vector perturbation (VP) precoding [3][4] are then proposed and can approach the performance of DPC. On the other hand, linear precoding schemes that require much less computational complexity have received increasing research attention. Among linear schemes, the zero-forcing (ZF) precoding scheme in [5] requires the least complexity, while its performance is far from DPC and the non-linear schemes. A regularized zero-forcing (RZF) scheme is then proposed in [6] to improve the rate performance of $\mathrm{ZF}$, especially at low signal-to-noise ratio (SNR) regime. A correlation rotation linear scheme is further proposed in [7] to offer additional performance gains by exploiting the constructive interference.

The above studies on precoding techniques are based on the assumption that conventional antenna arrays are employed at the transceivers, where each antenna element is connected to a dedicated radio frequency (RF) chain. Multiple RF chains will incur a large amount of hardware cost and consequent power consumption, which can be a limitation especially in the future energy-efficient communication systems. Furthermore, the spacing between adjacent antennas for conventional antenna arrays is usually designed larger than half of the wavelength to avoid the spatial correlation and mutual coupling effect, which is also a limitation for size-constrained devices such as small access points (APs). Toward this direction and to achieve energy-efficient transmission, an alternative compact antenna array, also known as electronically steerable parasitic array radiator (ESPAR), has been proposed with a much smaller antenna spacing [8][9]. Different from conventional MIMO arrays, ESPARs only require a few active antenna elements with RF chains, while other antenna elements are parasitic and excited passively by the mutual coupling effect. With a tunable load employed at each parasitic element, the mutual coupling and consequently the currents at each antenna port can be controlled to form the desired radiation patterns. With reduced number of RF chains, the hardware complexity and power consumption of the antenna array can be greatly alleviated, which makes ESPAR a space- and energy-efficient alternative to conventional arrays.

Due to the above advantages, ESPARs have received increasing research attention in recent years [10]-[15]. In [10][12], single-fed ESPARs are considered, where in [11] the calculation of each tunable load and the feeding voltage for the central element is given. In [12], it is shown that the currents at each antenna port of the ESPAR array can be considered as the input signals of the MIMO systems, which greatly simplifies the design of ESPARs. An optimizationbased scheme is proposed in [13] to obtain the values of each load subject to the input impedance requirement. Nevertheless, the above studies of ESPARs assume ideal and continuous load values, which is not feasible for current hardware technologies. In [14], a loading scheme is considered for ESPARs to support 16-QAM modulation, and the effect of impedance errors is simulated. In [15], we further consider the Gaussian random impedance errors for ESPARs, and mathematically analyze the effect of impedance errors, where it is shown that the presence of impedance errors results in an error floor at high SNR regime.

Instead of considering Gaussian random impedance errors, in this paper we focus on a more realistic ESPAR array where load impedances with quantized values are considered, and we study the precoding schemes with quantization errors. Noting that the presence of the impedance errors caused by quantization will lead to a performance loss especially at high SNR, we propose to approximate the currents of the ESPAR to the desired signals by optimization to minimize the performance degradation. We propose to jointly optimize the feeding voltages and each tunable load value, where it is 
further proven that any additional variations in the loads result in an additional noise term and the optimality is therefore achieved by optimizing the voltages only. The optimization problem can then be transformed into a convex optimization and can be efficiently solved. Specifically, when single-fed ESPARs are considered, we obtain the closed-form expression of the optimal feeding voltage. Numerical results show that the proposed quantization-robust scheme can compensate for the performance loss by quantization and better approach the performance of conventional MIMO systems, which enables the practical implementation of ESPARs.

Notations: $a$, a, and A denote scalar, vector and matrix, respectively. $\mathbb{E}\{\cdot\},(\cdot)^{*},(\cdot)^{H},(\cdot)^{-1}$, and $\operatorname{tr}\{\cdot\}$ denote expectation, conjugate, conjugate transpose, inverse and trace of a matrix respectively. $\|\cdot\|$ denotes the Frobenius norm, and $\mathbf{I}$ is the identity matrix. We denote $\mathbf{0}$ as a zero matrix or vector. $\mathcal{C}^{n \times n}$ represents an $n \times n$ matrix in the complex set, and $\operatorname{diag}(\cdot)$ denotes the conversion of a vector into a diagonal matrix with the values on its main diagonal. $\Re(\cdot)$ and $\Im(\cdot)$ denote the real and imaginary part of a complex number, respectively.

\section{SyStem MODEL}

This section introduces the signal model for the ESPAR array, followed by the channel modelling and quantization model in the load values.

\section{A. Signal Model}

We firstly consider the conventional array, and then extend to the ESPAR array. Assume a base station (BS) with $N_{t}$ transmit antennas, and each antenna is fed by an independent source with the complex voltage $v_{n}^{0}$. The equivalent circuit representation is shown in Fig. 1 (a), where $\mathbf{Z}_{m}^{0} \in \mathcal{C}^{N_{t} \times N_{t}}$ is the mutual impedance matrix that is related to the carrier frequency and antenna spacing. Then, the complex current vector $\mathbf{i}_{0}$ at the antenna port can be obtained based on the generalized Ohm's law as

$$
\mathbf{i}_{0}=\left[\operatorname{diag}\left(\mathbf{z}_{0}\right)+\mathbf{Z}_{m}^{0}\right]^{-1} \mathbf{v}_{0},
$$

where $\mathbf{v}_{0} \in \mathcal{C}^{N_{t} \times 1}$ denotes the voltage vector, and $\mathbf{z}_{0}=z_{0} \cdot \mathbf{I}$ is the output impedance vector. For a conventional MIMO array, $z_{0}$ is fixed and the desired signals are generated by adjusting $\mathbf{v}_{0}$. On the other hand, when an ESPAR-based array is considered, as shown in Fig. 1 (b), only the active $N$ antenna elements are fed with voltages, while the remaining $N_{t}-N$ elements are parasitic and each is employed with a tunable load. In this case, the current vector is obtained as

$$
\begin{aligned}
\mathbf{i} & =\left[\operatorname{diag}\left(\mathbf{z}_{L}\right)+\mathbf{Z}_{m}\right]^{-1}\left[v_{1}, \ldots, v_{N}, 0, \ldots, 0\right]^{T} \\
& =\left[\operatorname{diag}\left(\mathbf{z}_{L}\right)+\mathbf{Z}_{m}\right]^{-1} \mathbf{v}_{s} \\
& =\mathbf{Z}_{T} \mathbf{v}_{s} .
\end{aligned}
$$

In (2), $\mathbf{z}_{L}=\left[z_{0}, \ldots, z_{0}, z_{1}, \ldots, z_{N_{t}-N}\right]^{T}$ is the load impedance vector, where each load for the active $N$ elements is fixed as $z_{0}$ and each tunable load for the passive element is denoted as $z_{k}, k \in\left\{1,2, \ldots, N_{t}-N\right\} . \mathbf{Z}_{m}$ denotes the mutual impedance

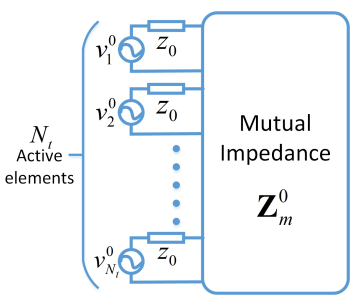

(a) Conventional array

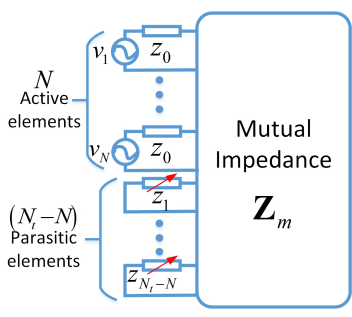

(b) ESPAR array
Fig. 1: Circuit representation

for the ESPAR array that is obtained from $\mathbf{Z}_{m}^{0}$ with some column re-arrangements. For simplicity we denote $\mathbf{Z}_{T}$ as the effective coupling matrix. It can be observed in (2) that different from conventional MIMO array where the currents are solely dependent on the voltage vector, for the ESPAR arrays the currents are jointly decided by both the voltage vector $\mathbf{v}_{s}$ and the load vector $\mathbf{z}_{L}$.

In this paper we consider a multi-user scenario, where the BS communicates with $K$ single-antenna users simultaneously. Following [12][13][15], the current vector can be considered as the system input of the ESPAR arrays, based on which a general system equation can be obtained as

$$
\mathbf{y}=\mathbf{H i}+\mathbf{n},
$$

where $\mathbf{y} \in \mathcal{C}^{K \times 1}$ is the received signal vector, and we denote $\mathbf{H} \in \mathcal{C}^{K \times N_{t}}$ as the channel matrix. $\mathbf{n} \in \mathcal{C}^{K \times 1}$ is the additive Gaussian noise vector and $\mathbf{n} \sim \mathcal{C N}\left(\mathbf{0}, \sigma^{2} \cdot \mathbf{I}\right)$, where the noise power is denoted as $\sigma^{2}$. In order to apply precoding schemes to ESPAR-based MIMO systems, the precoded signals are mapped to the currents at each antenna port [12][13]. For an ESPAR-based MU-MISO system, the current vector can then be expressed as

$$
\mathbf{i}=\frac{1}{f} \cdot \mathbf{P s},
$$

where $\mathbf{s} \in \mathcal{C}^{K \times 1}$ denotes the data symbol vector, the precoding matrix is denoted as $\mathbf{P} \in \mathcal{C}^{N_{t} \times K}$, and $f$ is the scaling factor to ensure that the signal power remains unchanged after precoding.

\section{B. Channel Model}

Since the antenna spacing for the ESPAR array is usually very small, the spatial correlation and mutual coupling effect should be considered in modelling the channel. Following [12][16] where it is shown and verified that conventional channel model can be applied to ESPAR-based arrays, in this paper we employ a geometric semi-correlated Rayleigh channel $\mathbf{H}=\left[\mathbf{h}_{1}^{T}, \ldots, \mathbf{h}_{K}^{T}\right]^{T}$, with each $\mathbf{h}_{k}$ expressed as

$$
\mathbf{h}_{k}=\mathbf{g}_{k} \mathbf{A}_{k} .
$$

In (5), $\mathbf{g}_{k} \in \mathcal{C}^{1 \times M}$ with $M$ being the number of directions of departure (DoDs), and each element in $\mathbf{g}_{k}$ follows the standard complex Gaussian distribution that forms the Rayleigh component of the channel. $\mathbf{A}_{k} \in \mathcal{C}^{M \times N_{t}}$ contains $M$ steering 
vectors at the transmitter side that form the correlation effect. For uniform linear arrays, as assumed in this paper, $\mathbf{A}_{k}$ is given by

$$
\mathbf{A}_{k}=\frac{1}{M}\left[\mathbf{a}^{T}\left(\theta_{k, 1}\right), \ldots, \mathbf{a}^{T}\left(\theta_{k, M}\right)\right]^{T},
$$

with each $\mathbf{a}\left(\theta_{k, m}\right) \in \mathcal{C}^{1 \times N_{t}}$ expressed as

$$
\mathbf{a}\left(\theta_{k, m}\right)=\left[1, e^{j 2 \pi d \sin \theta_{k, m}}, \ldots, e^{j 2 \pi\left(N_{t}-1\right) d \sin \theta_{k, m}}\right] .
$$

In (7), $d$ denotes the antenna spacing normalized by the carrier wavelength. $\theta_{k, m}$ denotes the angle of departure (AoD) and we assume each $\theta_{k, m}$ follows a uniform distribution in $[-\pi, \pi]$. We note that for the ESPAR array, since the mutual coupling effect has been included in the current vector $\mathbf{i}$ in (2), it is therefore not shown explicitly in the channel model.

\section{Parasitic Arrays with Quantized Loads}

In this paper we focus on the study of a more realistic ESPAR array model, where we consider the imperfection in the electronic components that only quantized load values with finite precision are feasible in practice [17]-[19]. With quantization, the load values of each tunable impedance can be expressed as

$$
\hat{z}_{k}=z_{k}+e_{k}, k \in\left\{1,2, \ldots, N_{t}-N\right\},
$$

where we denote $\hat{z}_{k}$ as the quantized load value for the $k$-th antenna, $z_{k}$ the desired load value, and $e_{k}$ the quantization error. Assuming that the quantization interval is $D$, we can express the potential values of each tunable load as

$$
\hat{z}_{k}=m_{k} D+j \cdot n_{k} D, m_{k}, n_{k} \in\{0, \pm 1, \pm 2, \ldots\} .
$$

\section{Proposed Quantization-Robust Scheme}

It has been shown in [14][15] that the presence of the errors in the load values greatly degrades the performance of the ESPAR array and results in an error floor at high SNR regime. Therefore in this section we propose to compensate for the performance loss by approximating the current vector with impedance errors to the desired current vector by optimization. Specifically, we propose to jointly optimize the feeding voltages $\mathbf{v}_{s}$ and each quantized load $\hat{z}_{k}$ such that the quantization effect is minimized.

In the presence of the quantized loads, we firstly rewrite (2) as

$$
\begin{aligned}
\hat{\mathbf{i}} & =\left[\operatorname{diag}\left(\hat{\mathbf{z}}_{L}\right)+\mathbf{Z}_{m}\right]^{-1} \mathbf{v}_{s} \\
& =\left[\operatorname{diag}\left(\mathbf{z}_{L}\right)+\mathbf{Z}_{m}+\mathbf{E}\right]^{-1} \mathbf{v}_{s},
\end{aligned}
$$

where $\hat{\mathbf{z}}_{L}$ denotes the quantized load vector. $\mathbf{E}=$ $\operatorname{diag}\left(\left[0, \ldots, 0, e_{1}, \ldots, e_{N_{t}-N}\right]\right)$ is the load error matrix, where we note that there exist quantization errors only for the tunable loads. Then, with the variations in the quantized loads and the voltages, the current vector with optimization can be expressed as

$$
\begin{aligned}
\mathbf{i}^{R} & =\left[\operatorname{diag}\left(\hat{\mathbf{z}}_{L}^{R}\right)+\mathbf{Z}_{m}\right]^{-1} \mathbf{v}_{s}^{R} \\
& =\left[\operatorname{diag}\left(\mathbf{z}_{L}\right)+\mathbf{Z}_{m}+\mathbf{E}^{R}\right]^{-1}\left(\mathbf{v}_{s}+\Delta \mathbf{v}\right) \\
& =\left[\operatorname{diag}\left(\mathbf{z}_{L}\right)+\mathbf{Z}_{m}+\mathbf{E}+D \cdot \operatorname{diag}(\mathbf{t})\right]^{-1}\left(\mathbf{v}_{s}+\Delta \mathbf{v}\right),
\end{aligned}
$$

where $\mathbf{i}^{R}$ is the optimized current vector, the optimized quantized loads are denoted as $\hat{\mathbf{z}}_{L}^{R}, \mathbf{v}_{s}^{R}$ denotes the optimized voltage vector and $\Delta \mathbf{v}$ is the variation in the feeding voltages. In (11), $\mathbf{E}^{R}=\mathbf{E}+D \cdot \operatorname{diag}(\mathbf{t}), \mathbf{t} \in \mathcal{C} \mathcal{Z}^{N_{t} \times 1}$ is the complex integer vector to be optimized that satisfies $\mathbf{t}(n)=0$, $n \in\{1, \ldots, N\}$, and $D \cdot \operatorname{diag}(\mathbf{t})$ then represents the additional quantized load values when optimality is reached. Based on (2), (11) can be further transformed into

$$
\begin{aligned}
& \mathbf{i}^{R} \\
= & {\left[\operatorname{diag}\left(\hat{\mathbf{z}}_{L}^{R}\right)+\mathbf{Z}_{m}\right]^{-1}\left\{\left[\operatorname{diag}\left(\hat{\mathbf{z}}_{L}\right)+\mathbf{Z}_{m}\right] \mathbf{i}+\Delta \mathbf{v}\right\} } \\
= & \mathbf{i}-\left[\operatorname{diag}\left(\hat{\mathbf{z}}_{L}^{R}\right)+\mathbf{Z}_{m}\right]^{-1} \mathbf{E}^{R} \mathbf{i}+\left[\operatorname{diag}\left(\hat{\mathbf{z}}_{L}^{R}\right)+\mathbf{Z}_{m}\right]^{-1} \Delta \mathbf{v} \\
= & \mathbf{i}+\left[\operatorname{diag}\left(\hat{\mathbf{z}}_{L}^{R}\right)+\mathbf{Z}_{m}\right]^{-1}\left(\Delta \mathbf{v}-\mathbf{E}^{R} \mathbf{i}\right) .
\end{aligned}
$$

Then, we can express the difference between the desired current vector and the optimized current vector as

$$
\begin{aligned}
\Delta \mathbf{i} & =\mathbf{i}^{R}-\mathbf{i} \\
& =\left[\operatorname{diag}\left(\hat{\mathbf{z}}_{L}^{R}\right)+\mathbf{Z}_{m}\right]^{-1}\left(\Delta \mathbf{v}-\mathbf{E}^{R} \mathbf{i}\right),
\end{aligned}
$$

which leads to the following proposition.

Proposition 1: $\Delta \mathbf{i}$ cannot be minimized to $\mathbf{0}$ by optimizing $\Delta \mathbf{v}$ that corresponds to the variations in the voltages and $\mathbf{t}$ that corresponds to the variations in the quantized load values.

Proof: To have $\Delta \mathbf{i}=\mathbf{0}$ is equivalent to

$$
\left[\operatorname{diag}\left(\hat{\mathbf{z}}_{L}^{R}\right)+\mathbf{Z}_{m}\right]^{-1}\left(\Delta \mathbf{v}-\mathbf{E}^{R} \mathbf{i}\right)=\mathbf{0} .
$$

Since $\Delta \mathbf{v}(n)=0, \forall n \in\left\{N+1, \ldots, N_{t}\right\}$, therefore we obtain that $\Delta \mathbf{v}-\mathbf{E}^{R} \mathbf{i} \neq \mathbf{0}$. Then, (14) is equivalent to having non-zero solutions for a linear system $\mathbf{U x}=\mathbf{0}$ with $\mathbf{U}=\left[\operatorname{diag}\left(\hat{\mathbf{z}}_{L}^{R}\right)+\mathbf{Z}_{m}\right]^{-1}$. Based on linear algebra theory, the following condition must be satisfied

$$
\operatorname{det}\left\{\left[\operatorname{diag}\left(\hat{\mathbf{z}}_{L}^{R}\right)+\mathbf{Z}_{m}\right]^{-1}\right\}=0 .
$$

(15) is not achievable for an inverse matrix, which completes the proof.

Proposition 1 implies that there always exists a performance loss compared to the conventional MIMO when quantized loads are employed. We then propose to minimize this performance gap by convex optimization, and the following proposition is given.

Proposition 2: When the optimality is achieved, $\mathbf{t}^{*}=\mathbf{0}$, which means that any further variations in the quantized loads will incur additional performance losses.

Proof: When there are only variations in the feeding voltages, we have $\Delta \mathbf{v} \neq \mathbf{0}$ and $\mathbf{t}=\mathbf{0}$. Then, based on (12) the current vector is obtained as

$\mathbf{i}^{R}=\mathbf{i}-\left[\operatorname{diag}\left(\hat{\mathbf{z}}_{L}^{R}\right)+\mathbf{Z}_{m}\right]^{-1} \mathbf{E i}+\left[\operatorname{diag}\left(\hat{\mathbf{z}}_{L}^{R}\right)+\mathbf{Z}_{m}\right]^{-1} \Delta \mathbf{v}$.

Then for the same voltages $\Delta \mathbf{v}$, when a variation in the quantized loads is further introduced, $\mathbf{t} \neq \mathbf{0}$ and we can further obtain

$$
\begin{aligned}
\mathbf{i}^{R}= & \mathbf{i}-\left[\operatorname{diag}\left(\hat{\mathbf{z}}_{L}^{R}\right)+\mathbf{Z}_{m}\right]^{-1} \mathbf{E i}+\left[\operatorname{diag}\left(\hat{\mathbf{z}}_{L}^{R}\right)+\mathbf{Z}_{m}\right]^{-1} \Delta \mathbf{v} \\
& -D \cdot\left[\operatorname{diag}\left(\hat{\mathbf{z}}_{L}^{R}\right)+\mathbf{Z}_{m}\right]^{-1} \operatorname{diag}(\mathbf{t}) \mathbf{i}
\end{aligned}
$$


Comparing (16) with (17), it is observed that an additional term is introduced in (17) with variations in the quantized loads, which contributes as an additional noise and increases the equivalent noise power. Therefore, to keep the noise power as low as possible, the optimal case is to keep the load values unchanged and only optimize the voltages.

We can then formulate the optimization problem as

$$
\begin{array}{ll}
\mathcal{P}_{1}: & \min _{\Delta \mathbf{v}}\|\Delta \mathbf{i}\|^{2} \\
\text { s.t. } & \Delta \mathbf{v}(n)=0, \forall n \in\left\{N+1, \ldots, N_{t}\right\}
\end{array}
$$

By denoting $\mathbf{P}=\left[\operatorname{diag}\left(\mathbf{z}_{L}\right)+\mathbf{Z}_{m}+\mathbf{E}\right]$ that is fixed with respect to $\Delta \mathbf{v}, \mathcal{P}_{1}$ can be transformed into a convex form as

$$
\begin{array}{ll}
\mathcal{P}_{2}: & \min _{\Delta \mathbf{v}}\left\|\mathbf{P}^{-1} \Delta \mathbf{v}-\mathbf{P}^{-1} \mathbf{E i}\right\|^{2} \\
\text { s.t. } & \Delta \mathbf{v}(n)=0, \forall n \in\left\{N+1, \ldots, N_{t}\right\}
\end{array}
$$

which can be efficiently solved by convex optimization tools such as CVX.

\section{A. Closed-Form Expression for Single-Fed ESPARs}

We further consider a special case where single-fed ESPARs are employed, and show that in this case a closed-form solution can be obtained. In the case of single-fed ESPARs, $N=1$, and by introducing an auxiliary complex variable $\alpha, \Delta \mathbf{v}$ can be expressed as

$$
\Delta \mathbf{v}=\alpha \cdot \mathbf{v}_{s}
$$

since there is only one entry in $\mathbf{v}_{s}$ that is non-zero for singlefed ESPARs. Then, based on (12) and the expression of $\mathbf{P}$, $\Delta \mathbf{i}$ for single-fed ESPARs can be expressed as

$$
\begin{aligned}
\Delta \mathbf{i} & =(1+\alpha) \mathbf{P}^{-1}(\mathbf{P}-\mathbf{E}) \mathbf{i}-\mathbf{i} \\
& =\left(\mathbf{I}-\mathbf{P}^{-1} \mathbf{E}\right) \mathbf{i} \cdot \alpha+\left[\left(\mathbf{I}-\mathbf{P}^{-1} \mathbf{E}\right)-\mathbf{I}\right] \mathbf{i} \\
& =\mathbf{Q} \cdot \alpha+(\mathbf{Q}-\mathbf{i}),
\end{aligned}
$$

where $\mathbf{Q}=\left(\mathbf{I}-\mathbf{P}^{-1} \mathbf{E}\right) \mathbf{i}$ and $\mathbf{Q} \in \mathcal{C}^{N_{t} \times 1}$. Then, $\|\Delta \mathbf{i}\|^{2}$ can be further expressed as

$$
\begin{aligned}
\|\Delta \mathbf{i}\|^{2}= & \operatorname{tr}\left\{\Delta \mathbf{i} \cdot \Delta \mathbf{i}^{H}\right\} \\
= & \operatorname{tr}\left\{[\mathbf{Q} \cdot \alpha+(\mathbf{Q}-\mathbf{i})][\mathbf{Q} \cdot \alpha+(\mathbf{Q}-\mathbf{i})]^{H}\right\} \\
= & \operatorname{tr}\left\{\mathbf{Q}^{H} \mathbf{Q} \cdot \alpha \alpha^{*}\right\}+2 \Re\left(\operatorname{tr}\left\{\left(\mathbf{Q} \mathbf{Q}^{H}-\mathbf{Q i}^{H}\right) \cdot \alpha\right\}\right) \\
& +\operatorname{tr}\left\{(\mathbf{Q}-\mathbf{i})(\mathbf{Q}-\mathbf{i})^{H}\right\}
\end{aligned}
$$

Since $\mathbf{Q}$ and $\mathbf{i}$ are independent of $\alpha$, the optimization for single-fed ESPAR arrays can be transformed into

$$
\mathcal{P}_{3}: \min _{\alpha} f(\alpha)
$$

where $f(\alpha)$ can be expressed as

$$
\begin{aligned}
f(\alpha) & =\operatorname{tr}\left\{\mathbf{Q}^{H} \mathbf{Q} \alpha \alpha^{*}\right\}+2 \Re\left(\operatorname{tr}\left\{\left(\mathbf{Q Q}^{H}-\mathbf{Q i}^{H}\right) \alpha\right\}\right) \\
& =\operatorname{tr}\left\{S \cdot \alpha \alpha^{*}\right\}+2 \Re(\operatorname{tr}\{\mathbf{T} \cdot \alpha\}) .
\end{aligned}
$$

In (24), $S$ and $\mathbf{T}$ are given by

$$
S=\mathbf{Q}^{H} \mathbf{Q}>0, \mathbf{T}=\mathbf{Q Q}^{H}-\mathbf{Q i}^{H} .
$$

By denoting

$$
\Re(\alpha)=\alpha_{R E}, \Im(\alpha)=\alpha_{I M},
$$

the objective function in $\mathcal{P}_{3}$ can be further obtained as

$$
\begin{aligned}
f(\alpha) & =\left\{S \alpha_{R E}^{2}+2 \sum_{i=1}^{N_{t}} \Re[\mathbf{T}(i, i)] \alpha_{R E}\right\} \\
& +\left\{S \alpha_{I M}^{2}-2 \sum_{i=1}^{N_{t}} \Im[\mathbf{T}(i, i)] \alpha_{I M}\right\} .
\end{aligned}
$$

It can be observed that the real part and imaginary part of $f(\alpha)$ are both in a quadratic form, and we can then obtain the optimal $\alpha^{*}$ as

$$
\alpha^{*}=\frac{-\sum_{i=1}^{N_{t}} \Re[\mathbf{T}(i, i)]}{S}+j \cdot \frac{\sum_{i=1}^{N_{t}} \Im[\mathbf{T}(i, i)]}{S} .
$$

With $\alpha^{*}$ obtained by (28), the optimal feeding voltage for single-fed ESPARs is obtained as

$$
\mathbf{v}_{s}^{R}=\left(1+\alpha^{*}\right) \mathbf{v}_{s} .
$$

\section{NumericAl Results}

To evaluate the performance of ESPAR-based MIMO systems and the proposed quantization-robust schemes, in this section we present numerical results based on Monte Carlo simulations. Perfect channel state information (CSI) is assumed throughout the simulations, and QPSK modulation is applied to evaluate the bit error rate (BER) performance. The simulation parameters are shown in Table I below.

\begin{tabular}{|c|c|}
\hline Simulation Parameters & ESPAR arrays \\
\hline Operating Frequency & $2.5 \mathrm{GHz}$ \\
\hline Antenna Spacing $d$ & $\lambda / 4$ \\
\hline Quantization Interval $D$ & 1 \\
\hline Number of DoDs $M$ & 50 \\
\hline Number of Transmit Antennas $N_{t}$ & 64 \\
\hline Number of Active Antennas $N$ & 7 \\
\hline Number of Parasitic Antennas $N_{t}-N$ & 57 \\
\hline Number of Users $K$ & 6 \\
\hline
\end{tabular}

TABLE I: Simulation Parameters

For simplicity we employ ZF precoding scheme at the transmitter, which leads to the expression of the desired current vector as

$$
\mathbf{i}=\frac{1}{f} \cdot \mathbf{H}^{H}\left(\mathbf{H} \mathbf{H}^{H}\right)^{-1} \mathbf{s},
$$

while the performance gains of the proposed quantizationrobust scheme trivially apply to other precoding schemes. For clarity, the following abbreviations are applied: 'MIMO ZF' denotes the conventional MIMO systems with ZF precoding; 'ESPAR ZF ideal' denotes the ESPAR-based MIMO systems with ideally continuous loads; 'ESPAR ZF Quantized' denotes ESPARs with quantized loads; 'ESPAR ZF Robust' denotes the proposed quantization-robust scheme. 
In Fig. 2, the BER performance of multiple-fed ESPARs is shown with respect to the transmit SNR, where it is observed that the ideal ESPAR array can achieve similar performance to the conventional MIMO systems, while the presence of the quantization in the load values severely degrades the detection performance. With the proposed quantization-robust scheme, the performance loss can be greatly alleviated and the ESPARs with quantized loads can better approach the performance of conventional MIMO systems, which enables the implementation of the ESPAR arrays in practice.

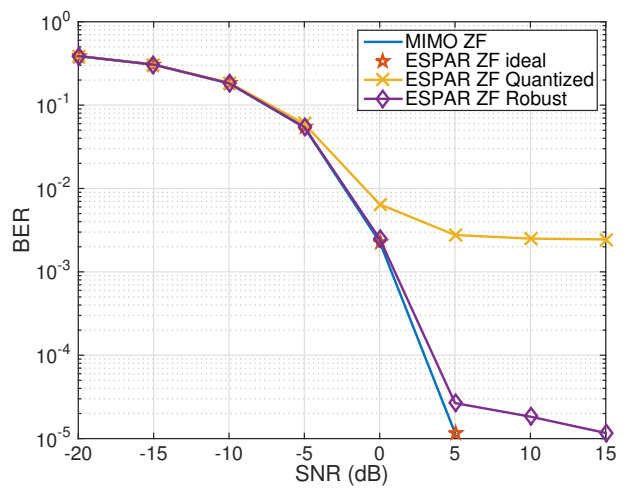

Fig. 2: BER vs transmit SNR, $N_{t}=64, N=7, K=6, D=1$, QPSK

A similar result can be observed in Fig. 3 where we compare the BER with the increasing quantization interval $D$, where it is observed that the proposed quantization-robust scheme is less sensitive to the increase in the quantization interval, which validates its robustness.

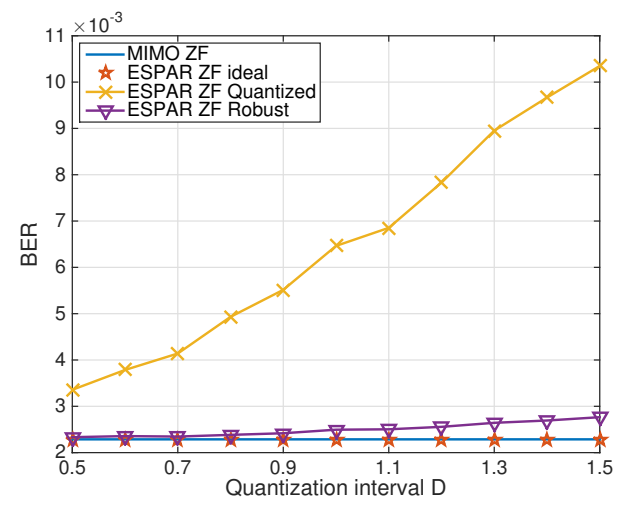

Fig. 3: BER vs quantization interval $D, N_{t}=64, N=7, K=6$, $\mathrm{SNR}=0 \mathrm{~dB}$, QPSK

\section{CONCLUSION}

In this paper, the precoding schemes for practical ESPARs with quantized loads are studied. The quantization in the loads is shown to result in a performance loss, especially at high SNR regime. We then propose to optimize the feeding voltages and each quantized load to alleviate the performance loss, where it is shown that the optimality is reached by optimizing the voltages only. Specifically, we obtain the closed-form solution when single-fed ESPARs are considered. Numerical results observe a significant performance gain for the proposed quantization-robust scheme over conventional ESPARs with quantized loads.

\section{ACKNOWLEDGMENT}

This work was supported by the Royal Academy of Engineering, U.K., the Engineering and Physical Sciences Research Council (EPSRC) project EP/M014150/1, and the China Scholarship Council (CSC).

\section{REFERENCES}

[1] M. Costa, "Writing on Dirty Paper," IEEE Trans. Inf. Theory, vol. IT-29, no. 3, pp. 439-441, May 1983.

[2] A. Garcia-Rodriguez and C. Masouros, "Power-Efficient TomlinsonHarashima Precoding for the Downlink of Multi-User MISO Systems," IEEE Trans. Commun., vol. 62, no. 6, pp. 1884-1896, June 2014.

[3] B. M. Hochwald, C. B. Peel, and A. L. Swindlehurst, "A VectorPerturbation Technique for Near-Capacity Multiantenna Multiuser Communication-Part II: Perturbation," IEEE Trans. Commun., vol. 53, no. 3, pp. 537-544, March 2005.

[4] A. Li and C. Masouros, "A Constellation Scaling Approach to Vector Perturbation for Adaptive Modulation in MU-MIMO," IEEE Wireless Commun. Lett., vol. 4, no. 3, pp. 289-292, March 2015.

[5] T. Haustein, C. von Helmolt, E. Jorswieck, V. Jungnickel, and V. Pohl, "Performance of MIMO Systems with Channel Inversion," in Vehicular Technology Conference. IEEE 55th Vehicular Technology Conference. VTC Spring 2002 (Cat. No.02CH37367), vol. 1, 2002, pp. 35-39.

[6] C. B. Peel, B. M. Hochwald, and A. L. Swindlehurst, "A VectorPerturbation Technique for Near-Capacity Multiantenna Multiuser Communication-Part I: Channel Inversion and Regularization," IEEE Trans. Commun., vol. 53, no. 1, pp. 195-202, Jan. 2005.

[7] C. Masouros, "Correlation Rotation Linear Precoding for MIMO Broadcast Communications," IEEE Trans. Sig. Process., vol. 59, no. 1, pp. 252-262, Jan. 2011.

[8] K. Gyoda and T. Ohira, "Design of Electronically Steerable Passive Array Radiator (ESPAR) Antennas," in IEEE Antennas and Propagation Society International Symposium, vol. 2, Salt Lake City, UT, USA, 2000, pp. 922-925.

[9] A. Kalis, A. G. Kanatas, and C. B. Papadias, Parasitic Antenna Arrays for Wireless MIMO Systems. Springer, 2014.

[10] H.-T. Liu, S. Gao, and T.-H. Loh, "Electronically Small and Low Cost Smart Antenna for Wireless Communication," IEEE Trans. Ant. Propag., vol. 60, no. 3, pp. 1540-1549, March 2012.

[11] V. I. Barousis and C. B. Papadias, "Arbitrary Precoding with SingleFed Parasitic Arrays: Closed-Form Expression and Design Guidelines," IEEE Wireless Commun. Lett., vol. 3, no. 2, pp. 229-232, Apr. 2014.

[12] G. C. Alexandropoulos, V. I. Barousis, and C. B. Papadias, "Precoding for Multiuser MIMO Systems with Single-Fed Parasitic Antenna Arrays," in 2014 IEEE Global Communications Conference, Austin, TX, 2014, pp. 3897-3902.

[13] L. Zhou, F. A. Khan, T. Ratnarajah, and C. B. Papadias, "Achieving Arbitrary Signals Transmission Using a Single Radio Frequency Chain," IEEE Trans. Commun., vol. 63, no. 12, pp. 4865-4878, Oct. 2015.

[14] B. Han, V. I. Barousis, C. B. Papadias, A. Kalis, and R. Prasad, "MIMO over ESPAR with 16-QAM Modulation," IEEE Wireless Commun. Lett., vol. 2, no. 6, pp. 687-690, Dec. 2013.

[15] A. Li and C. Masouros, "Performance Analysis for Single-Fed ESPAR in the Presence of Impedance Errors and Imperfect CSI," in 2016 IEEE International Conference on Communications (ICC), Kuala Lumpur, 2016, pp. 1-6.

[16] O. N. Alrabadi, C. Divarathne, P. Tragas, A. Kalis, N. Marchetti, C. B. Papadias, and R. Prasad, "Spatial Multiplexing with a Single Radio: Proof-of-Concept Experiments in an Indoor Environment with a 2.6GHz Prototype," IEEE Commun. Lett., vol. 15, no. 2, pp. 178-180, Feb. 2011.

[17] D. S. Holder, Ed., Electrical Impedance Tomography: Methods, History and Applications. CRC Press, Dec. 2004.

[18] S. L. Carson, M. E. Orazem, O. D. Crisalle, and L. Garcia-Rubio, "On the Error Structure of Impedance Measurements - Simulation of FRA Instrumentation," Journal of the Electrochemical Society, vol. 150, no. 10 , pp. 477-490, 2003.

[19] — "On the Error Structure of Impedance Measurements - Series Expansions," Journal of the Electrochemical Society, vol. 150, no. 10, pp. 501-511, 2003. 